\title{
Dynamic responses of sunspots to their ambient magnetic configuration
}

\author{
S. P. Bagare \\ Indian Institute of Astrophysics, Bangalore, India \\ email: bagare@iiap.res.in
}

\begin{abstract}
In our earlier study of a revisit of the classic Wilson Effect, it was found that a large proportion of sunspots do not display the geometric effect which is ascribed to a depression of the umbra. It was shown that the presence or absence of the effect, observed close to the limb, depends upon the ambient magnetic configuration of the sunspot. In this follow up study, we look for the impact of changes in ambient magnetic configuration on the measurable properties of sunspots during their disk passage, using observations obtained at the Kodaikanal observatory during 1978-80. Digitized photoheliogram data were used to examine and measure areas of spots and their umbrae for 101 cases. Magnetic field measures published by the Academy of sciences, Leningrad were used to evaluate the ambient magnetic configuration. The results indicate that the extent of magnetic bipolarity is associated with changes in the proportion of the area of the penumbra to that of the umbra. In regular spots, the relative area of penumbra reduces with reduction in the strength of ambient opposite polarity spots and pores. However, in the presence of pore sized blob(s) in penumbra, and with associated emerging fluxes, the penumbra is significantly enlarged. But in the presence of a light bridge or a split umbra, the relative area of penumbra is considerably reduced.
\end{abstract}

Keywords. photosphere, sunspots, magnetic fields

\section{Introduction}

One of the early observations of the physical properties of sunspots was that single isolated sunspots, as they approach the solar limb, display a reduction of the width of the penumbra on the disk-center side more rapidly than the width on the limb-ward side. This geometric effect, known as the Wilson effect, is understood to be due to a depression of the umbra with respect to surrounding photosphere, in the range of 400-800 km (Gokhale \& Zwaan 1972), and the increased transparency of the sunspot atmosphere owing to its low temperature and gas pressure (Thomas \& Weiss 2008). Many extensive studies have, however, shown that a large proportion of sunspots either do not display the effect or show an inverse effect, but they were generally ignored as being attributable to nonuniformity in the shape of penumbrae or various difficulties in the actual measurement of the effect (McIntosh, P. S. 1981).

Bagare \& Gupta (1998) concluded from a study of 20 sunspots that the ambient magnetic configuration appears to play a key role in the occurrence or absence of the classic Wilson effect. Bagare (2010) studied 580 epochs of 253 sunspots located within a longitude zone of $40^{\circ}$ to $80^{\circ}$ on either side of the central meridian and concluded that all the unipolar sunspots of magnetic class $\alpha, \alpha p$, or $\alpha f$ invariably display the classic Wilson effect while spots of magnetic class $\beta, \beta p$ or $\beta f$ do not display the Wilson effect, and spots of class $\beta \gamma$ and $\gamma$ display an inverse effect. It was also shown that the occurrence of inverse effect was invariably associated with the presence of mixed polarity pores in the neighbourhood of the main spot. 
Our case studies in the meantime suggested that various properties of sunspots probably depend upon the magnetic class of the sunspot. The properties noted were the changing location in penumbra of the peak velocity in Evershed flows, the presence or absence of a weak bright ring around the spot, the domination of three or five minute intensity oscillations, and various properties of umbral dots. Since these results indicated that the ambient magnetic configuration appears to have an observable impact on the sunspots, the study of a significant number of sunspots during their disk passage was taken up to evaluate their ambient magnetic configurations and to measure the areas of their umbrae and penumbrae, and thereby to look for possible correlations.

Further, the results reported by many authors, especially those using X-ray images of the corona and the extreme ultraviolet observations with TRACE spacecraft, show that the field lines that are less steeply inclined in penumbrae rise up to form loops that extend for great distances across the solar surface, connecting either to other sunspots or to distant footpoints (apparently those of opposite magnetic polarity). These results have been summarized by Thomas \& Weiss (2008).

\section{Obsrevation and Measurements}

The Kodaikanal photoheliogram and spectroheliogram digitization program is going on for the past many years. CCD cameras (4k x $4 \mathrm{k}$ ) are being used with high precision lab spheres and light tables for the digitization. Photoheliograms digitized for the years 1978-80 were used for the present study. The raw images in Fits format were processed for dark subtraction and flat fielding. Measurements of the full spot and umbral areas were carried out on IDL platform using the XROI program. The boundaries marked manually agreed well with the intensity values of $59 \%$ and $85 \%$ of the quiet photosphere for umbra-penumbra and penumbra-photosphere boundaries respectively, as adopted by Brandt, Schmidt, \& Steinegger (1990). Only the spots with clearly measurable umbral and penumbral boundaries were selected for measurement. Spots of all magnetic categories were included. The measured areas, converted to millionths of solar hemisphere, are accurate to within a few percent.

The values of longitudinal component of magnetic field were obtained from the monthly Solar Data Bulletins (SDB) published by the Academy of Sciences, Leningrad, which provide daily sketches and magnetic field strengths of all sunspots and strong pores. Our earlier measurements of Zeeman splittings and the longitudinal field strengths at Kodaikanal agreed closely with the values reported in SDB. The extent of Zeeman splitting provides estimates accurate only to a few hundred Gauss and measurements are not made for the weak pores. There is also a time lag of about three hours in the observations used in SDB for evaluation but the ambient magnetic configuration, as well as the reported field strengths, remain comparable. These accuracies may be considered sufficient for the purpose of this study.

In order to represent the ambient magnetic configuration of sunspots, we define a magnetic bipolarity index as follows:

$$
Q_{s}=\sum\left(B_{i} / d_{i}\right)+\sum 4\left(N_{j} / d_{j}\right)
$$

where $B_{i}$ are the magnetic field strengths of the opposite polarity sunspots and strong pores, in hundred Gauss measures, at heliographic distances of $d_{i}$ from the sunspot, taken 


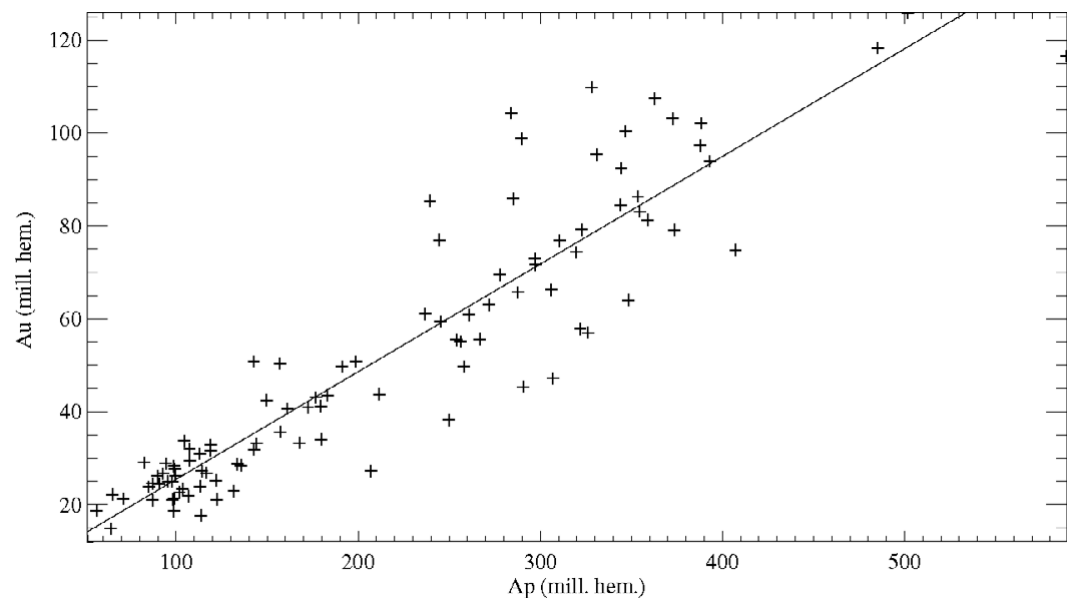

Figure 1. Area of umbra vs the area of penumbra for 101 spots, in millionths of visible hemisphere. A spread in the area of penumbra for a given area of umbra is cognizable.

up to a maximum distance of about 40 degrees. The heliographic distance of 40 degrees was chosen since it was observed in our earlier study that spots and pores at this distance appear to have an impact on the Wilson effect in sunspots. $N_{j}$ is the number of pores at a group distance of $d_{j}$ from the sunspot. $N_{j}$ accounts for the pores for which the field strengths are not given in SDB. A mean field strength of 800 Gauss ( 8 in hundred Gauss measures) is assumed. It is also assumed that the pores emerge in pairs and hence the factor $8 / 2=4$ per pair is used in representing the opposite polarity pores. Pores within a heliographic distance of about $15^{0}$ only are included. These measurements were carried out for 101 cases observed during the period 1978-80, corresponding to the maximum phase of solar cycle 21, providing a large number of active sunspot groups for the study.

\section{Results and discussion}

The areas of the entire spot $\left(A_{s}\right)$, the umbra alone $\left(A_{u}\right)$, and that of the penumbra alone $\left(A_{p}\right)$, in millionths of the visible hemisphere, were computed for all the spots studied. A plot of $A_{u}$ vs $A_{p}$ is shown in Figure 1. A linear relationship between the parameters is evident, though with a significant spread in the values of $A_{p}$ for any given value of $A_{u}$. A histogram of the ratio of $A_{u}$ to that of $A_{s}$ is shown in Figure 2. It is readily seen that majority of the sunspots have the area ratio in the range of 0.17 to 0.23 , in general agreement with the earlier studies by Brandt, Schmidt, \& Steinegger (1990) and Ringnes, T. S. (1964). However, we do find a significant number of spots in the lower range of 0.13 to 0.17 as well as in the higher range of 0.23 to 0.27 .

Further, in Figure 3 the ratio of $A_{p}$ to $A_{u}$ is plotted against the magnetic bipolarity index $Q_{s}$, estimated using Equation 2.1. It is evident that the area of penumbra varies largely over a wide range of 2.7 to about 6.5 times that of the umbra. We classifed the spots into three major morphological categories and studied the properties of individual sunspots in each category, looking for possible patterns in the dynamic behaviour. The results of this analysis are discussed below.

\subsection{Penumbral area in regular spots}

A study of the distribution of the spots in Figure 3, in the light of their magnetic type, reveals the following characteristics. The spots represented with asterisks are regular 


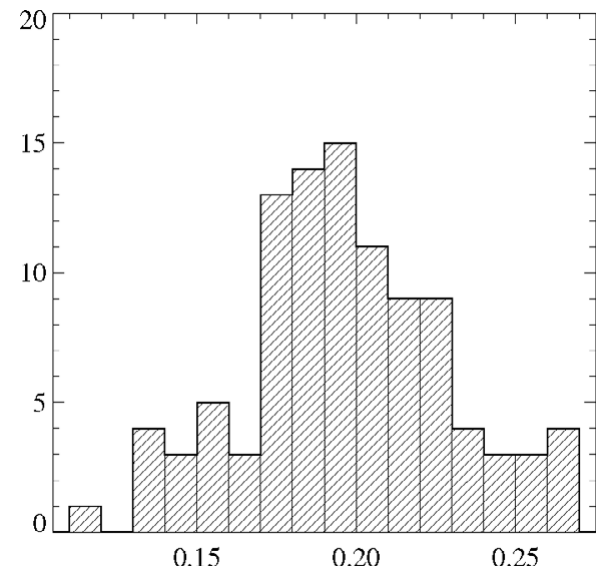

Figure 2. Distribution of the ratio of the area of umbra to the area of spot.

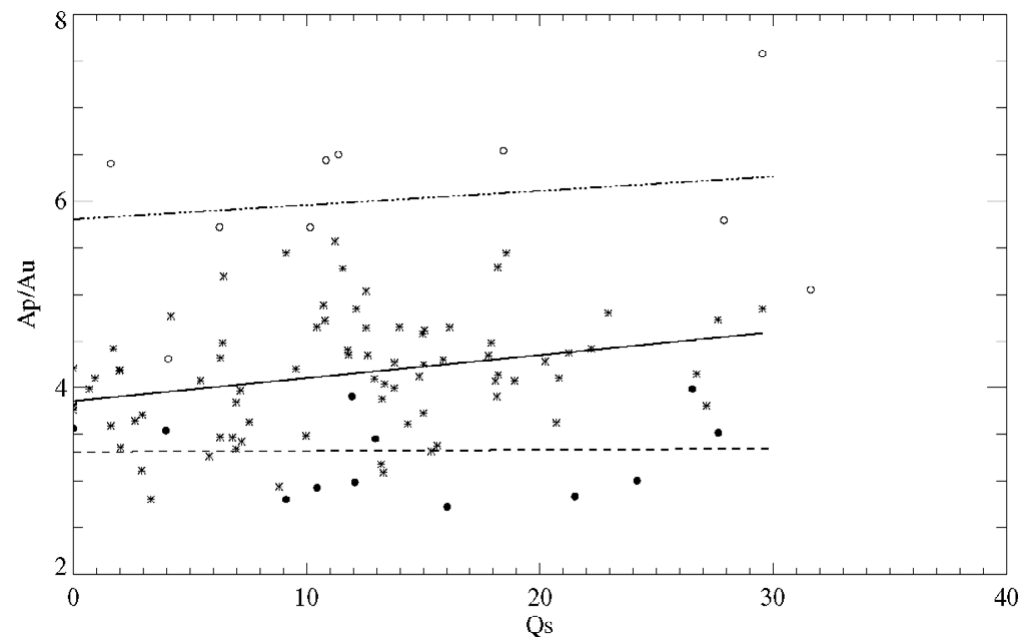

Figure 3. Ratio of area of penumbra to that of the umbra vs the magnetic bipolarity index defined by equation 2.1. Asterisks represent the regular spots, hollow circles represent spots having pore sized blob(s) in their penumbrae, and solid circles represent spots having light bridges or split umbrae. Regression fits are plotted for these using solid line, dash and dots line, and dashed line respectively.

spots having neither pore-sized blobs in their penumbrae nor having any evident light bridges or split umbrae. These spots show a clear increase in the value of $A_{p} / A_{u}$ with increasing value of $Q_{s}$, the range of $A_{p} / A_{u}$ being about 2.7 to 5.6 . In general, single isolated spots of $\alpha$ type, which also have low values of $Q_{s}$ say below 10, have low values for $A_{p} / A_{u}$. On the other hand, spots of class $\beta \gamma$ and $\gamma$, which are generally associated with large number of pores, apparently of mixed polarity, have high values of $Q_{s}$ and relatively higher values of $A_{p} / A_{u}$. The spots of $\beta$ type have intermediate values for both $Q_{s}$ and $A_{p} / A_{u}$. A regression fit for these regular spots is shown with a solid line in the Figure. In the light of known properties of birth, evolution, and decay of sunspots, it may be inferred from this result that spots in the early stages of formation of the group begin with their position close to higher end of the solid line and as they transform into single 
isolated spots, they approach the lower end of the line in the plot, implying a significant reduction in the relative areas of penumbrae.

\subsection{Penumbral area in spots associated with flux emergence}

We separately examined the properties of sunspots which have one or more (upto four) pore sized blobs in their penumbrae. It may be recalled that such blobs are found predominantly during the early phases of sunspot groups which have a large number of mixed polarity pores in the vicinity (Bray \& Loughhead (1964)), representing flux emergence in the region. These spots are represented with hollow circles in Figure 3. A line of dash and dots is regression fitted to these points. It may be noticed that the slope of this fit is comparable to that for the regular spots. The values of $A_{p} / A_{u}$ for these spots are very significantly increased, by about $50 \%$ on the average, with respect to the regular spots. Some of the related properties observed are as follows;

(a) The blobs have intensities which are between those of the umbra and the penumbra.

(b) The blobs are normally present on the side of the spot which is closer to accompanying pores.

(c) The increase in penumbral area occurs on the side containing the blob(s).

(d) Contribution of the blobs themselves to the increase in the area of penumbra is about 5 to $6 \%$ for one to three blobs. In the case of a spot having four blobs, the contribution is about $17 \%$.

(e) Majority of the spots which have low values of $Q_{s}$ and possess blob(s) in penumbra, display a large number of accompanying pores within the following 24 hours. This indicates that the emerging flux was already present at the time of observation and mergers were taking place.

$(f)$ For the spot type discussed in $e$ above, the contribution of the blobs to the increase in area of penumbra is only about 1.5 to $5 \%$.

Further, Schlichenmaier et al.(2010) have reported the observation of formation of a sunspot penumbra. They find that while the area of the umbra remains intact, the flux emergence in the vicinity is followed by merger of like polarity end of the bipole with the spot and an increase in the area of the penumbra. In the case of spots studied by us above and found to possess highly enlarged penumbrae, the penumbrae are already well developed but it appears that the process of flux merger is still in progress. Once the flux mergers are complete, that is when pore sized blobs are no more present in penumbra, the spot apparently settles down to its regular ratio of $A_{p} / A_{u}$.

\subsection{Penumbral area in spots having light bridges or split umbrae}

Another category we studied is that of spots having light bridges or split umbrae. These spots are represented with filled circles in Figure 3. The relative areas of penumbrae in these spots is less by about 12 to $27 \%$ with respect to those of the regular spots and they do not change practically over the entire range of $Q_{s}$ values. It turns out that out of the 12 cases studied, 11 are spots in old active regions, in their second or third rotations and are fast approaching the phase of being a unipolar spot, followed by further decay. The lower its $Q_{s}$ value, the closer is the spot to its transition to unipolar phase, 2 to 3 days for $Q_{s}$ below 10 and 5 to 6 days for the rest (with the exception of one spot which decays faster). In the remaining one case out of 12 , the spot has a light bridge in its young and complex phase. However, this too displays a low value of $A_{p} / A_{u}$. It may be summarized that in sunspots having light bridges or having split or nearly split umbrae, the relative area of 
penumbra is significantly decreased, as if the penumbra has shrunk in size. Curiously, this property appears to be independent of the ambient magnetic configuration.

\section{Conclusion}

It is evident from the study that the sunspot penumbra responds dynamically to ambient magnetic configuration. In regular sunspots, the ratio of the area of penumbra to that of the umbra shows an increase with increasing collective strength of opposite polarity spots and pores in the vicinity. In the presence of mixed polarity pores, implying flux emergence in the region, the increase in relative area of penumbra is the highest. This is exactly the phase when the spot shows an inverse Wilson effect if it is close to the limb. Therefore, if we assume that the extended penumbra is also elevated higher than in its normal phase, the inverse Wilson effect observed under these conditoins can perhaps be understood better.

In the presence of flux emergence in the form of pores and with pore sized blob(s) present in it, the penumbra is very significantly enlarged, by upto $50 \%$ on average, particularly in the direction of the blob and nearby pores. In many cases where only the blob is present, the accompanying mixed polarity pores show up within the following day implying that the emerging flux was present at the time of observation and the penumbra was responding to it. Therefore, there is a clear indication that spots have highly enlarged penumbrae in their formative and flux merger phase, and as they evolve to the status of a single isolated spot, the relative areas of their penumbrae decrease steadily and considerably.

Another aspect noted in this study is that in the presence of a light bridge and also when the umbra is in the process of splitting or is just split, the relative area of penumbra is significantly reduced implying that the penumbra has shrunk from its regular size. The relative area of penumbra in this phase is independent of the ambient magnetic configuration. It therefore appears that the penumbra is attempting to retain the spot together against attempts by convective processes to split it into fragments.

It emerges from this study that the relative area of penumbra plays a fundamental role in the stability of sunspots. It may be hypothesised that the spot responds to changes in ambient magnetic configuration and to the attempts to fragment it, through a balancing of magnetic and gas pressures. These responses are apparently readily reflected by changes in the relative area of the penumbra.

\section{Acknowledgement}

The data used in this study was taken from the Kodaikanal Observatory archives and digitized under the digitization program of IIA. The author's participation in the symposium was made possible with support from the IAU, IIA, and NASA-NSF funds.

\section{References}

Bagare, S. P. 2010, in: Hasan, S. S., \& Rutten, R. J.(eds.) Magnetic Coupling between the Interior and Atmosphere of the Sun, p 398

Bagare, S. P. \& Gupta, S. S. 1998, Bull. Astron. Soc. India, 26, 197

Brandt, P. N., Schimdt, W., \& Steinegger, M. 1990, Solar Phys., 129, 191

Bray, R. J. \& Loughhead, R. E. 1964, in The International Astrophysics Series, Chapman \& Hall, London Sunspots

Gokhale, M. H. \& Zwaan, C. 1972, Solar Phys., 26, 52 
McIntosh, P. S. 1981, in: Cram, L. E., \& Thomas, J. H. (eds.), Sacramento Peak Observatory The Physics of Sunspots

Ringnes, T. S. 1964, Astrophys. Norvegica, 8, 303

Schlichenmaier, R., Bello Gonzalez, N., Rezaei, R., \& Waldmann, T. A. 2010, Astron. Nachr. 331,563

Thomas, J. H. \& Weiss, N. O. 2008, in: Cambridge Astrophysics Series 46 Sunspots and Starspots (Cambridge University Press) 